\title{
Der DIEP Lappen als Standard in der autologen Brustrekonstruktion - Ergebnisse und Algorithmus zur erfolgreichen Rekonstruktion
}

\author{
The DIEP Flap as Method of Choice in Breast Reconstruction - Results and Protocol \\ for Succesful Reconstruction
}

Autoren

Institut
M. Otte, C. Andree, M. Hagouan, P. Richrath, A. Abu-Ghazaleh, B. Munder

Plastische und Ästhetische Chirurgie, SANA Klinik Gerresheim, Düsseldorf

\author{
Schlüsselwörter \\ tion \\ - DIEP Lappenplastik \\ - MS-2-TRAM \\ freie Lappenplastik \\ - Algorithmus \\ Key words \\ autologous breast \\ reconstruction \\ - free flap \\ - DIEP flap \\ - MS-2-TRAM \\ - protocol
}

- Autologe Brustrekonstruk- eingereicht $\quad 31.10 .2014$ akzeptiert 13.1.2015

Bibliografie

DOI http://dx.doi.org/ 10.1055/s-0034-1398662 Handchir Mikrochir Plast Chir 2015; 47: 222-227 (c) Georg Thieme Verlag KG Stuttgart · New York ISSN 0722-1819

Korrespondenzadresse Dr. med. Maximilian Otte, MD Plastische und Ästhetische Chirurgie

SANA Klinik Gerresheim Gräulingerstraße 120 40625 Düsseldorf maximilian.otte@sana.de

\section{Zusammenfassung}

V

Hintergrund: Die autologe Brustrekonstruktion gewinnt zunehmend an Bedeutung insbesondere vor dem Hintergrund der erhöhten Raten an prophylaktischen Mastektomien bei BRCA 1-3 Mutationen. Bezüglich der Indikation und Komplikationsrate zwischen freien DIEP und TRAM Lappenplastiken zur autologen Brustrekonstruktion existieren heterogene Daten.

Material und Methoden: Zwischen Januar 2010 und Dezember 2011 erhielten 362 Patientinnen eine autologe Brustrekonstruktion mittels freier DIEP oder MS-2-TRAM Lappenplastik. Die Wahl der Lappenplastik zwischen DIEP und MS-2-TRAM erfolgte standardisiert durch einen Algorithmus mit präoperativer CT-Angiografie, intraoperativer Lappenperfusionskontrolle und Dopplerdetektion. Die Auswertung berücksichtigte Lappenrandnekrosen <20\%, Lappenrandnekrosen $>20 \%$ und komplette Lappenverluste, Fettnekrosen sowie operative Anastomosenrevisionen.

Ergebnisse: Es wurden bei 362 Patienten 419 freie Lappenplastiken zur Brustrekonstruktion durchgeführt (366 DIEP und 53 MS-2-TRAM). Bei 66 Patientinnen erfolgte eine bilaterale Rekonstruktion (52 Doppel DIEPs, 9 DIEP und MS-2TRAMS sowie 5 Doppel MS-2-TRAMS). Die totale Lappenverlustrate betrug insgesamt 0,24\%. Lappenrandnekrosen $<20 \%$ zeigten sich bei $0,72 \%$ (DIEP 0,82\%, MS-2-TRAM 0\%), Lappenrandnekrosen $>20 \%$ bei $0,72 \%$ (DIEP 0,69\% MS 2 TRAM $0,98 \%$ ). Bei einer DIEP Lappenplastik kam es zu einem Totalverlust. Die Rate der Anastomosenrevisionen zeigte sich mit insgesamt $0,48 \%$ gering. Es zeigte sich keine signifikant erhöhte Komplikationsrate in einer der Gruppen. Nach Einführung des Algorithmus reduzierte sich der Anteil der MS-2-Tram Lappenplastiken im Vergleich zu dem Zeitraum davor um ca. $20 \%$ auf Werte zwischen 10,9-13,5\% aller Brustrekonstruktionen.

\section{Abstract \\ $\nabla$}

Background: Autologous breast reconstruction is becoming increasingly important, especially in light of the increased rates of prophylactic mastectomies with BRCA mutations. Regarding the indications and complications between free TRAM and free DIEP flaps for autologous breast reconstruction the current data is not clear.

Materials and Methods: All patients who received an autologous breast reconstruction between January 2010 and January 2014 using free DIEP or free MS-2-TRAM flaps were included in the study. The choice of flap between DIEP and MS-2-TRAM was performed by a standardised algorithm with preoperative CT angiography, intraoperative evaluation of the flap perfusion and Doppler detection. The analysis took into account partial flap necrosis $<20 \%$ and $>20 \%$ ), complete flap loss, flap necrosis and surgical revision of the anastomosis.

Results: The study considered 362 women who received a total of 419 free flaps for breast reconstruction. 66 patients received a bilateral reconstruction (52 double DIEPS, 9 DIEP and MS2 TRAM and 5 double MS2 TRAMs). The total flap loss was $0.24 \%$. Partial necrosis $<20 \%$ occurred in $0.72 \%$ (DIEP $0.82 \%$, MS2 TRAM 0\%), partial necrosis $>20 \%$ in $0.72 \%$ (DIEP $0.69 \%$ MS2 TRAM $0.98 \%)$. One DIEP flap was lost $(0.24 \%)$. After implementing our protocol the rate of MS-2-Tram flaps could be reduced to $10-15 \%$ of all autologous breast reconstruction procedures as compared to the years before.

Conclusion: Through the application of the presented algorithm for breast reconstruction with free DIEP flap, such surgery is a safe treatment option if it is done at high frequency in a microsurgical centre. Flap loss and complications are not increased compared to MS2 TRAM flaps. The decision for the selection of the appropriate flap for breast reconstruction should be based on 
Schlussfolgerung: Durch die Anwendung des vorgestellten Algorithmus ist die Brustrekonstruktion mit freier DIEP Lappenplastik eine sichere Therapieoption wenn sie hochfrequent in einem mikrochirurgischen Zentrum erfolgt. Die Lappenverlustund Komplikationsrate ist im Vergleich zu MS-2-Tram nicht erhöht. Die Entscheidung zur Selektion der geeigneten Lappenplastik sollte anhand des vorgestellten Algorithmus erfolgen um ein sicheres reproduzierbares Ergebnis zu erzielen.

\section{Einleitung \\ $\nabla$}

Brustkrebs ist weiterhin der häufigste Tumor der Frau und resultiert in bis zu 30\% der Fälle in einer Mastektomie. Auch die Möglichkeit der Gentestung bzgl. familiärer Dispositionen führt zu einer erhöhten Rate an prophylaktischen Mastektomien [1]. Die Brustrekonstruktion mit autologem Gewebe, insbesondere nach Bestrahlung, ist mittlerweile als Goldstandard anzusehen. Im Verlauf der letzten Jahrzehnte wurden verschiedene Möglichkeiten der autologen Brustrekonstruktion beschrieben. Von den Anfängen der gestielten Latissimus dorsi Lappenplastik bis zur Entwicklung von komplexen freien Perforator basierten Lappenplastiken, ist hier insbesondere der DIEP Lappen hervorzuheben [2-6]. Zunächst von Koshima beschrieben, hat er sich mittlerweile als favorisiertes Verfahren in der autologen Brustrekonstruktion etabliert [7-9]. Der Anteil der DIEP Lappenplastik in der autologen Brustrekonstruktion ist zwischen 2009 und 2011 in den USA signifikant angestiegen und erreicht mittlerweile fast den gleichen Anteil wie gestielte TRAM und Latissimus dorsi Lappenplastiken [10].

Es bestehen kontroverse Ansichten bzgl. der Zuverlässigkeit der freien DIEP Lappenplastik im Vergleich zur freien TRAM Lappenplastik. Insbesondere bei erhöhtem BMI oder Komorbiditäten wie Diabetes oder Nikotinkonsum sind die beschriebenen Erfolgsraten variabel [11-13]. Die DIEP Lappenplastik ist im Vergleich zur gestielten und freien TRAM Lappenplastik häufig mit erhöhten venösen Abflussstörungen sowie einer erhöhten Lappenverlustrate assoziiert $[12,14,15]$. Häufig wird hier der DIEP mit dem klassischen freien TRAM und nicht mit dem MS-2TRAM verglichen, der durch eine Präservation der Rectus abdominis Muskulatur eine deutlich verringerte Hebedefektmorbidität aufweist [16,17].

Mehrere Autoren haben bisher Algorithmen beschrieben, die die intraoperative Lappenselektion sowie Therapie von Perfusionsstörungen beinhalten [18,19]. Lindsey hat bereits 2007 durch die Anwendung eines intraoperativen Algorithmus die Erfolgsrate seiner DIEP und MS-2-TRAM Lappenplastiken von 92 auf $100 \%$ steigern können. Jedoch erfolgte hier keine präoperative CT Angiografie [20]. Bezüglich der präoperativen CT Angiografie konnte gezeigt werden, dass hiermit eine zuverlässige Darstellung der Perforansgefäße und deren intramuskulärem Verlauf möglich ist [21]. Auch eine signifikannte Reduktion der Präparationszeit von DIEP Lappenplastiken bei standardmäßiger, präoperativer CT Angiografie wurde berichtet [22].

Eine standardisierter Algorithmus ist zur sicheren Durchführung der autologen Brustrekonstruktion unabdingbar. Seit Januar 2010 wird ein solcher Algorithmus in unserer Klinik eingesetzt. Ziel der Studie ist der Vergleich unserer Komplikationen von freien DIEP und MS-2-TRAM Lappenplastiken zur Brustrekonstruktion zwischen 01/2010 und 12/2011. the presented algorithm in order to achieve secure and reproducible results.

\section{Patienten, Material und Methoden}

Im Rahmen einer Single Center Studie erfolgte eine retrospektive Analyse von allen Patientinnen die zwischen Januar 2010 und Dezember 2011 eine autologe Brustrekonstruktion mittels freier DIEP oder MS-2-TRAM Lappenplastik erhielten. Die Wahl der Lappenplastik zwischen DIEP und MS-2-TRAM erfolgte standardisiert durch einen Algorithmus aus präoperativer CT Angiografie, intraoperativer Lappenperfusionskontrolle und Dopplerdetektion. Die Auswertung berücksichtigte Teilnekrosen unter $20 \%$, Teilnekrosen über $20 \%$, komplette Lappenverluste und Fettnekrosen sowie operative Anastomosenrevisionen. Alle Patientinnen wurden standardisiert 6 Monate postoperativ elektiv einbestellt.

\section{Präoperative CT Angiografie}

Ab Januar 2010 erfolgte bei allen Patienten präoperativ eine CT Angiografie zur Darstellung der Perfusionsverhältnisse der Bauchdecke und Planung des operativen Prozedere. Alle CT Angiografien wurden in dem gleichen radiologischen Institut mit einem Philips Brilliance ICT Gerät durchgeführt. Die Untersuchung umfasste das Areal $5 \mathrm{~cm}$ oberhalb des Umbillicus bis zur Symphyse. Alle Patienten erhielten einen intravenösen Bolus Kontrastmittel (Ultraviss 370 Bayer). Die Bildakquirierung erfolgte in 0,6mm Schichten. Daraufhin wurden die MIP Schichten in $5 \mathrm{~mm}$ Dicke und $10 \mathrm{~mm}$ Dicke mit einem jeweiligen Schichtabstand von $5 \mathrm{~mm}$ in axialer und sagittaler Orientierung rekonstruiert. Die Gefäßrekonstruktion erfolgte durch Volume Rendering an der Auswerterkonsole durch einen erfahrenen Radiologen.

\section{Präparation und Perforatorselektion}

Der Operateur evaluiert präoperativ die Aufnahmen der CT Angiografie und markiert die Hauptperforatoren, die vom Radiologen in den Schichten markiert worden sind in Relation zum Bauchnabel an der liegenden Patientin.

Die Präparation der Empfängergefäße sowie der Lappenplastik erfolgt in 2 Teams und ausschließlich mit bipolarer Diathermie. Nach Identifikation des dominanten Perforators durch den Operateur werden alle übrigen Perforatoren temporär mit Bulldogklemmen abgeklemmt. Je nachdem wie viele Perforatoren für die Perfusion der Lappenplastik benötigt werden erfolgt zu diesem Zeitpunkt die Entscheidung zwischen DIEP oder MS-2TRAM.

Hauptentscheidungskriterien für die Wahl eines MS-2-TRAMS gegenüber einer DIEP Lappenplastik sind sowohl die Perforatoranatomie als auch die Perfusionsverhältnisse der Lappenplastik. Bei der intra-operativen klinischen Evaluation zeigte sich in unserem Kollektiv bei Raucherinnen, die präoperativ eine Nikotinkarenz nicht einhalten konnten, häufiger eine arterielle Minderperfusion als bei Nicht-Raucherinnen oder Raucherinnen, die präoperative eine Nikotinkarenz einhielten. Hier kann die 
Notwendigkeit bestehen, eine MS-2-TRAM Lappenplastik zu bevorzugen.

Adäquate Perforatoren zeigen eine gute Pulsation nach Darstellung und Dopplerkontrolle. Der Durchmesser beträgt mind. $1 \mathrm{~mm}$, die Begleitvene zeigt sich mit gutem venösen Rückfluss und der intramuskuläre Verlauf ist kurz. Zeigt der Perforator keinen adäquaten Durchmesser bei fraglicher Perfusion kann die Perfusion einer kompletten DIEP Lappenplastik nicht auf diesem beruhen.

Die Perfusion der DIEP Lappenplastik wird im Allgemeinen nach der von Hartrampf et al. beschriebenen Zonen eingeteilt [23].

Die Perfusion intraoperativ muss sowohl arteriell als auch venös evaluiert werden. In unserer Praxis erfolgt das sowohl klinisch durch visuelle Kontrolle der Rekapillarisierung, Inspektion der Blutungen aus dem Rand der Lappenplastik sowie Dopplerdetektion des arteriellen und venösen Schenkels des Lappenstiels. Auch wenn in überwiegender Anzahl der Komplikationen eine venöse Abflussstörung vorliegt, kann die arterielle Minderperfusion zu ausgeprägten Fettnekrosen führen [24,25].

Nach dem Absetzen der Lappenplastik wird diese durch ein kaltes, feuchtes Bauchtuch gekühlt. Die Anastomosen werden von Hand genäht und mit Fibrinkleber versiegelt. Nach Re-Perfusion wird die Lappenplastik durch warme Bauchtücher gewärmt. Sollte kein adäquater venöser Abfluss bestehen werden beide Lappenvenen angeschlossen oder evtl. noch zusätzlich die initial präparierte SIEV Vene angeschlossen.

\section{Postoperatives Management}

Das in unsere Klinik übliche intra- und postoperative Management wurde bereits 2010 von Langer et al. im Detail beschrieben und weicht seitdem nicht davon ab [19].

Vor Freigabe der Anastomosen erhalten die Patientinnen 2500 IE Heparin, postoperativ $100 \mathrm{mg}$ Aspirin p.o. für 6 Wochen und niedermolekulares Heparin für die Dauer der Immobilisation zur Thromboseprophylaxe. Perioperativ erhalten die Patientinnen eine Antibiotikaprophylaxe mi $3 \times 2$ gr. Cefazolin i.v./24h.

\section{Ergebnisse}

$\nabla$

Es wurden bei 362 Patienten insgesamt 419 freie Lappenplastiken zur Brustrekonstruktion durchgeführt (366 DIEP und 53 MS-2-TRAM). Bei 66 Patientinnen erfolgte eine bilaterale Rekonstruktion (52 Doppel DIEPs, 9 DIEP und MS-2-TRAMS sowie 5 Doppel MS-2-TRAMS). Die totale Lappenverlustrate betrug insgesamt $0,24 \%$. Lappenrandnekrosen $<20 \%$ zeigten sich bei $0,72 \%$ (DIEP 0,82\%, MS-2-TRAM 0\%), Lappenrandnekrosen $>20 \%$ bei 0,72\% (DIEP 0,69\% MS 2 TRAM 0,98\%). Die Rate der Anastomosenrevisionen zeigte sich mit insgesamt $0,48 \%$ gering. Fettgewebsnekrosen traten bei $1,91 \%$ der Patientinnen, die eine Rekonstruktion mittels DIEP Erhielten auf. Es zeigte sich keine signifikant erhöhte Komplikationsrate in einer der Gruppen (० Tab. 1).

Randnekrosen sind unterteilt in Lappenrandnekrosen von über $20 \%$ und unter $20 \%$ der Fläche der Lappenplastik. Fettgewebsnekrosen sind davon zu unterscheiden. Eine Revision aufgrund eines Hämatoms im Bereich der Empfängerstelle war in dem Studienzeitraum bei 3 Patienten die eine DIEP Lappenplastik erhielten indiziert ( $0,82 \%$ aller DIEP Lappenplastiken).

Wenn diese Ergebnisse mit den Daten der Jahre davor verglichen werden, zeigt sich, dass sich durch die Einführung der CT Angiografie die durchgeführten Lappenplastiken deutlich zugunsten

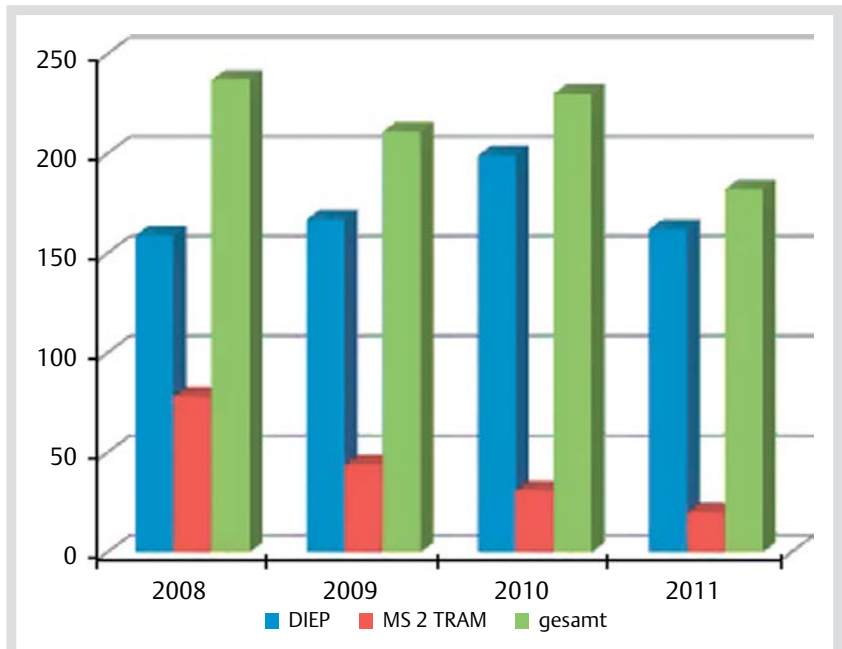

Abb. 1 Anteil von DIEP und MS 2 TRAM an durchgeführten Brustrekonstruktionen vor und nach Etablierung des Algorithmus mit CT Angiografie die seit 2010 standardmäßig präoperativ erfolgt. Der Anteil der MS-2-TRAM Lappenplastiken konnte von 32,9\% 2008 auf 11,05\% 2012 reduziert werden.

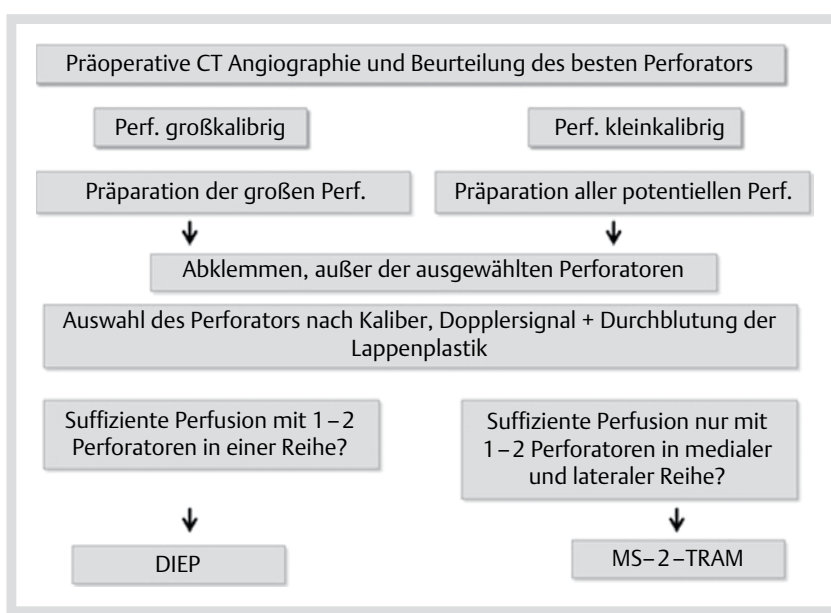

Abb. 2 Algorithmus zur Selektion der geeigneten Lappenplastik anhand prä- und intraoperativer Evaluation.

des DIEPs verschoben haben $\odot$ Tab. 1. Der Anteil der MS 2 TRAM Lappenplastiken liegt nach 2010 zwischen 10 und 15\% ( $\bullet$ Abb. 1-4).

\section{Diskussion \\ $\nabla$}

Unsere Ergebnisse zeigen, dass durch den eingesetzten Algorithmus in unserem Zentrum eine deutliche Verschiebung der autologen Brustrekonstruktionen zur DIEP Lappenplastik erfolgte. Der Anteil der MS-2-TRAM Lappenplastiken an den autologen Brustrekonstruktionen konnte um fast $20 \%$ reduziert werden, da durch die präoperative CT Angiografie eine bessere Planung möglich gewesen ist.

Durch die präoperative CT Angiografie können die Hauptperforatoren identifiziert werden und so die Präparation intraoperativ auf diese fokussiert werden. Somit können kleinere Perforatoren bei der Präparation direkt ligiert oder geklippt werden. Hierdurch entfällt ein Präparieren von kleineren Perforatoren was zu einer Verkürzung der OP Zeit beiträgt. 
In einem von Wurzer et al. kürzlich erschienenen Artikel wird in einem sehr kleinen Kollektiv mit n=30 ein Vorteil der Komplikationsrate zugunsten des TRAM Lappens beschrieben. Die Autoren ziehen bei Komorbiditäten den freien MS-2-TRAM der DIEP Lappenplastik vor. Eine solche Vorgehensweise wiederspricht wegweisenden Publikationen mit hohen Fallzahlen [16,17]. Unsere Ergebnisse zeigen, dass die Komplikationsrate durch einen DIEP im Vergleich zum freien MS-2-TRAM nicht erhöht ist und die Entscheidung zur Durchführung einer MS-2-TRAM Lappenplastik in Zusammenschau der präoperativen Diagnostik und intraoperativen Perfusions-verhältnisse und nicht anhand von Komorbiditäten zu erfolgen hat [26].

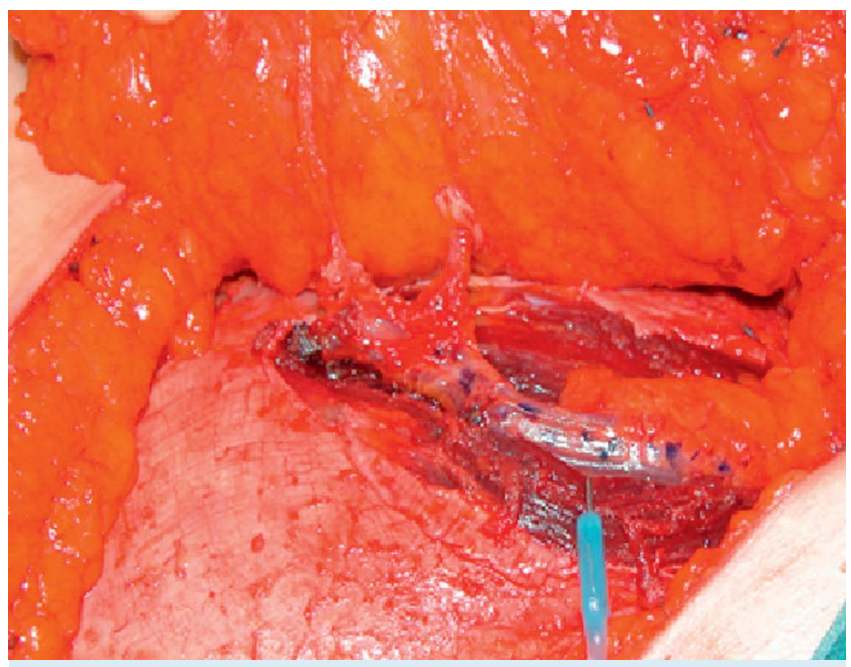

Abb. 3 Gute Perfusion der Lappenplastik nur durch mehrere Perforatoren aus medialer Reihe, Entscheidung für MS-2-TRAM.

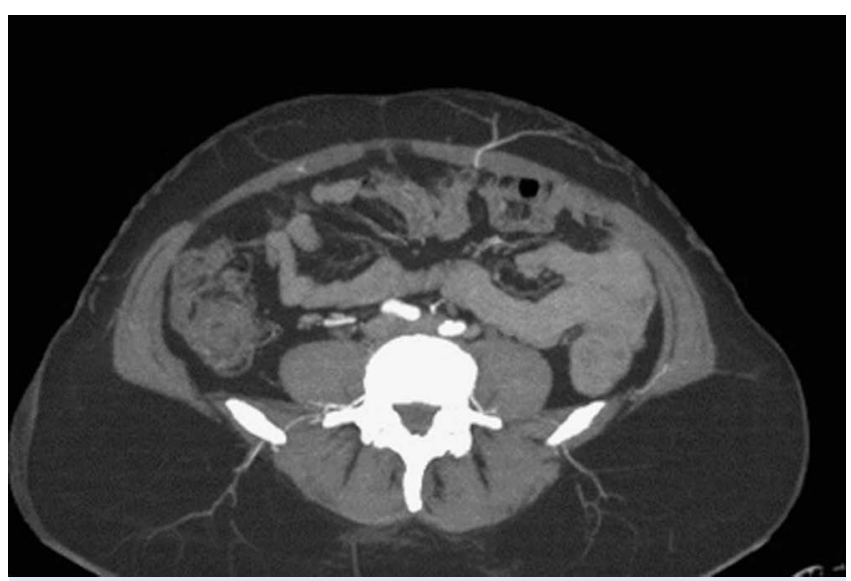

Abb. 4 Präoperative CT Angiografie, Identifikation eines großkalibrigen Perforators.
Wie in der Arbeit von Lindsey et al beschrieben kann durch Anwendung eines Algorithmus eine adäquate Perforator Selektion erfolgen und die Rate der MS-2-TRAMS deutlich gesenkt werden [20]. In unserem Zentrum wird dieser Algorithmus seit Januar 2010 mit standardisierter präoperativer CT Angio angewendet. Dadurch kam es in unserem Kollektiv zu einer deutlichen Verschiebung der Lappenplastiken zugunsten des DIEPs. Nahabedian et al. sahen bei 177 Patientinnen keinen Unterschied bezüglich Lappenverlustrate, Fettnekrose oder venöse Abflussstörungen zwischen DIEP und MS-2-Tram. Ein Vorteil des DIEPS bzgl. besserer Bauchwandstärke bei bilateraler Rekonstruktion stellte sich jedoch deutlich heraus [16]. Dies entspricht unseren Erfahrungen, weshalb alle Patientinnen primär für eine DIEP Lappenplastik geplant werden und die Entscheidung zur Durchführung eines MS-2-TRAMS nur intraoperativ anhand der Lappendurchblutung erfolgt.

Die Perfusion der DIEP Lappenplastik wird im Allgemeinen nach der von Hartrampf et al. beschriebenen Zonen eingeteilt [23]. In Arbeiten von Holm konnte gezeigt werden, dass die DIEP Lappenplastik eher als in der Mittellinie geteilte Lappenplastik, mit einem axialen Perfusionsmuster auf der ipsilateralen und einem Random pattern Perfusionsmuster auf der kontralateralen Seite, gesehen werden sollten [27]. In unserer Erfahrung zeigt sich, dass wie von Wong et al. beschrieben, die medialen Perforatoren größerer Anteile der Lappenplastik perfundieren und sich eher an der Hartrampf Einteilung orientieren, während laterale Perforatoren eher von der Perfusion her an der Holms Einteilung orientiert sind [28].

Die Rate an Fettnekrosen ist bei freien TRAM Lappenplastiken mit bis zu 6,9\% und bei freien DIEP Lappenplastiken sogar bei bis zu 14,4\% beschrieben. In einer Metaanalyse von Khansa et al. konnten als prädiktive Faktoren für Fettnekrosen Adipositas, prä- und postrekonstruktive Radiatio, Nikotinkonsum und präoperativ vorhandene Narben ausgemacht werden [25]. Garvey et al. sahen keinen Vorteil des freien Trams gegenüber Diep bzgl. Fettnekrosen nach Bestrahlung bei einzeitiger Brustrekonstruktion [29]. 2011 konnten Seidenstücker et al. bei 624 Patienten die eine freie Brustrekonstruktion erhielten, zeigen, dass bei Komorbiditäten wie Adipositas und Nikotinkonsum die Komplikationen erhöht sind, die Brustrekonstruktion mit autologem DIEP und MS-2-TRAM jedoch ein sicheres Verfahren ist [12].

Man et al. sahen in einer Metaanalyse von $n=1171$ dass der DIEP weniger zuverlässig ist als der TRAM bzgl. Nekrosen und Lappenverlustrate [17]. Dies widerspricht unseren Ergebnissen, da wir bei 419 freien Lappenplastiken keine erhöhte Komplikationsrate bei den DIEP Lappenplastiken feststellen konnte. Im Vergleich zu anderen mikrochirurgischen Zentren ist die intraoperative Antikoagulation mit 2500 IE Heparin hoch gewählt, es kam jedoch mit 0,82\% aller DIEP Lappenplastiken zu keiner erhöhten Rate an revisionspflichtigen Hämatomen im Bereich der Empfängerstelle.

Tab. 1 Brustrekonstruktionen mit freien DIEP und MS 2 TRAM Lappenplastiken zwischen 01/2010 und $12 / 2011$.

\begin{tabular}{|c|c|c|c|c|c|}
\hline Lappentyp & Anzahl & Randnekrosen $<20 \%$ & Randnekrosen $>20 \%$ & $\begin{array}{l}\text { kompletter } \\
\text { Lappenverlust }\end{array}$ & $\begin{array}{l}\text { Operative } \\
\text { Anastomosenrevision }\end{array}$ \\
\hline DIEP & $366(87,35 \%)$ & $12(3,28 \%)$ & $3(0,82 \%)$ & $1(0,27 \%)$ & $2(0,55 \%)$ \\
\hline MS 2 Tram & $53(12,65 \%)$ & $2(3,77 \%)$ & $0(0 \%)$ & $0(0 \%)$ & $0(0 \%)$ \\
\hline gesamt & 419 & $14(3,34 \%)$ & $3(0,72 \%)$ & $1(0,24 \%)$ & $2(0,48 \%)$ \\
\hline Doppel DIEP & $52(12,41 \%)$ & $1(1,92 \%)$ & $1(1,92 \%)$ & $0(0 \%)$ & $1(1,92 \%)$ \\
\hline Dopple MS2 Tram & $5(1,19 \%)$ & $0(0 \%)$ & $0(0 \%)$ & $0(0 \%)$ & $0(0 \%)$ \\
\hline DIEP+MS 2 Tram & $9(2,15 \%)$ & $1(0 \%)$ & $0(0 \%)$ & $0(0 \%)$ & $1(11,11 \%)$ \\
\hline
\end{tabular}


Eine standardisierter Algorithmus ist zur sicheren Durchführung der autologen Brustrekonstruktion unabdingbar. Die präoperative CT Angiografie kann hier eine große Hilfe bzgl. der Auswahl des geeigneten Hauptperforators sein, jedoch kann der dominante Perforator nur adäquat intraoperativ beurteilt werden, da die Auswahl sonst dem reinen Zufall überlassen ist. Unter Beibehaltung eines standardisierten Vorgehens zeigen sich keine Unterschiede bzgl. perfusionsbedingte Komplikationen zwischen freiem MS-2-TRAM und DIEP [30].

\section{Schlussfolgerung}

Durch die Anwendung des vorgestellten Algorithmus ist die Brustrekonstruktion mit freier DIEP Lappenplastik eine sichere Therapieoption wenn sie hochfrequent in einem mikrochirurgischen Zentrum erfolgt. Die Lappenverlust- und Komplikationsrate des DIEPS ist im Vergleich zu MS-2-Tram nicht erhöht. Die Entscheidung zur Selektion der geeigneten Lappenplastik sollte anhand des vorgestellten Algorithmus erfolgen um ein sicheres, reproduzierbares Ergebnis zu erzielen.

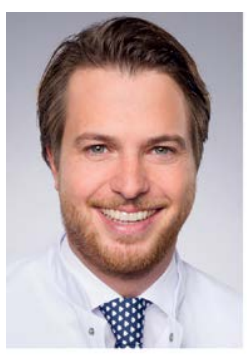

Maximilian Otte

Geb. am 21.12.1981. Medizinstudium an der Universität Ulm und Heidelberg mit Auslandsaufenthalten in der University of the West Indies Barbados sowie University of Southern California Los Angeles.

Er arbeitete über 5 Jahre an der BG Unfallklinik Ludwigshafen in der Klinik für Hand-, Plastische und Rekonstruktive Chirurgie und promovierte 2012 über „Management und Outcome von Patienten mit strominduzierten Verbrennungen Retrospektive Analyse von 128 Fällen“. 2014 absolvierte er ein 3-monatiges Fellowship in ästhetischer plastischer Chirurgie bei Prof. Per Heden in den Akademikliniken Stockholm. Seit dem 01.09.2014 ist er in der Klinik für Plastische und Ästhetische Chirurgie bei Prof. Andree in den SANA Kliniken Düsseldorf tätig.

Interessenkonflikt: Nein

\section{Literatur}

1 Society AC. American Cancer Society. Cancer facts and Figures 2013. Atlanta

2 Olivari $N$. The latissimus flap. Br J Plast Surg 1976; 29: 126-128 Available from: http://www.ncbi.nlm.nih.gov/pubmed/776304

3 Allen RJ, Tucker C. Superior gluteal artery perforator free flap for breast reconstruction. Plast Reconstr Surg 1995; 95: 1207-1212 Available from: http://www.ncbi.nlm.nih.gov/pubmed/15988253

4 Allen RJ, Levine JL, Granzow JW. The in-the-crease inferior gluteal artery perforator flap for breast reconstruction. Plast Reconstr Surg 2006; 118: 333-339 Available from: http://www.ncbi.nlm.nih.gov/ pubmed $/ 16874198$

5 Allen RJ, Haddock NT, Ahn CY et al. Breast reconstruction with the profunda artery perforator flap. Plast Reconstr Surg 2012; 129: 16e-23e Available from http://www.ncbi.nlm.nih.gov/pubmed/22186541

6 Pelzer M, Reichenberger Ma, Germann G. Microsurgical techniques for breast reconstruction. Chirurg 2011; 82: 807-812 Available from: http://www.ncbi.nlm.nih.gov/pubmed/21845484
7 Koshima I, Soeda S. Inferior epigastric artery skin flaps without rectus abdominis muscle. Br J Plast Surg 1989; 42: 645-648 Available from: http://www.ncbi.nlm.nih.gov/pubmed/2605399

8 Allen $R$, Treece $P$. Deep inferior epigastric perforator flap for breast reconstruction. Ann Plast Surg 1994 Available from: http://journals. lww.com/annalsplasticsurgery/Abstract/1994/01000/Deep_Inferior_ Epigastric_Perforator_Flap_for.7.aspx

9 Healy C, Allen RJ. The evolution of perforator flap breast reconstruction: twenty years after the first DIEP flap. J Reconstr Microsurg 2014; 30: 121-125 Available from: http://www.ncbi.nlm.nih.gov/ pubmed/24163223

10 Pien I, Caccavale S, Cheung MC et al. Evolving Trends in Autologous Breast Reconstruction: Is the Deep Inferior Epigastric Artery Perforator Flap Taking Over? Ann Plast Surg 201400 Available from: http:// www.ncbi.nlm.nih.gov/pubmed/25180959

11 Nahabedian MY, Momen B, Galdino G et al. Breast Reconstruction with the free TRAM or DIEP flap: patient selection, choice of flap, and outcome. Plast Reconstr Surg 2002; 110: 466-475 discussion 476-477 Available from: http://www.ncbi.nlm.nih.gov/pubmed/12142662

12 Seidenstuecker K, Munder B, Mahajan AL et al. Morbidity of microsurgical breast reconstruction in patients with comorbid conditions. Plast Reconstr Surg 2011; 127: 1086-1092 Available from: http://www. ncbi.nlm.nih.gov/pubmed/21364411

13 Tran NV, Buchel EW, Convery P. a. Microvascular complications of DIEP flaps. Plast Reconstr Surg 2007; 119: 1397-1405 discussion 1406-1408 Available from: http://www.ncbi.nlm.nih.gov/pubmed/17415232

14 Blondeel PN, Arnstein M, Verstraete $K$ et al. Venous congestion and blood flow in free transverse rectus abdominis myocutaneous and deep inferior epigastric perforator flaps. Plast Reconstr Surg 2000; 106: 1295-1299 Available from: http://www.ncbi.nlm.nih.gov/pubmed/11083558

15 Garvey PB, Buchel EW, Pockaj Ba et al. DIEP and pedicled TRAM flaps: a comparison of outcomes. Plast Reconstr Surg 2006; 117: 1711-1719 discussion 1720-1721 Available from http://www.ncbi.nlm.nih.gov/ pubmed/16651940

16 Nahabedian MY, Tsangaris T, Momen B. Breast Reconstruction with the DIEP Flap or the Muscle-Sparing (MS-2) Free TRAM Flap: Is There a Difference? Plast Reconstr Surg 2005; 115: 436-444 Available from: http://content.wkhealth.com/linkback/openurl?sid=WKPTLP: landingpage \&an $=00006534-200502000-00010$

17 Man L-X, Selber JC, Serletti JM. Abdominal wall following free TRAM or DIEP flap reconstruction: a meta-analysis and critical review. Plast Reconstr Surg 2009; 124: 752-764 Available from: http://www.ncbi. nlm.nih.gov/pubmed/19342994

18 Galanis C, Nguyen P, Koh J et al. Microvascular lifeboats: a stepwise approach to intraoperative venous congestion in DIEP flap breast reconstruction. Plast Reconstr Surg 2014; 134: 20-27 Available from: http://www.ncbi.nlm.nih.gov/pubmed/25028816

19 Langer S, Munder B, Seidenstuecker K et al. Development of a surgical algorithm and optimized management of complications - based on a review of 706 abdominal free flaps for breast reconstruction. Med Sci Monit 2010; 16: CR518-CR522 Available from: http://www.ncbi. nlm.nih.gov/pubmed/20980954

20 Lindsey JT. Integrating the DIEP and muscle-sparing (MS-2) free TRAM techniques optimizes surgical outcomes: presentation of an algorithm for microsurgical breast reconstruction based on perforator anatomy. Plast Reconstr Surg 2007; 119: 18-27 Available from: http://www. ncbi.nlm.nih.gov/pubmed/17255652

21 Fansa H, Schirmer S, Frerichs $O$ et al. Stellenwert der CT-Angiografie der Bauchwand für die Planung und Operation von DIEP-, TRAMund SIEA-Lappenplastiken. Handchir Mikrochir plast Chir 2011; 43: 81-87 Available from: https://www.thieme-connect.com/products/ ejournals/abstract/10.1055/s-0030-1262844

22 Kuekrek H, Paepke S, Dobritz M et al. Präoperative CT Angiogriafie zur Planung freier Perforans Lappenplastiken(DIEP Flaps) zur Brustrekonstruktion. Handchir Mikrochir plast Chir 2011; 43: 88-94

23 Hartrampf $C$. Breast reconstruction with a transverse abdominal island flap. Plast Reconstr 1982 Available from: http://journals.lww. com/plasreconsurg/Abstract/1982/02000/Breast_Reconstruction_ with_a_Transverse_Abdominal.6.aspx

24 Lie KH, Barker AS, Ashton MW. A classification system for partial and complete DIEP flap necrosis based on a review of 17,096 DIEP flaps in 693 articles including analysis of 152 total flap failures. Plast Reconstr Surg 2013; 132: 1401-1408 Available from: http://www.ncbi.nlm. nih.gov/pubmed/24281570 
25 Khansa I, Momoh AO, Patel PP et al. Fat necrosis in autologous abdomen-based breast reconstruction: a systematic review. Plast Reconstr Surg 2013; 131: 443-452 Available from: http://www.ncbi.nlm.nih. gov/pubmed/23446559

26 Wurzer P, Spendel S. Besteht ein Unterschied zwischen DIEP-und freiem TRAM-Lappen aus physiologischer und psychologischer Sicht? Eine retrospektive Patientenbefragung. Handchirurgie •...2014;256-262 Available from: https://www.thieme-connect.com/products/ejournals/ abstract/10.1055/s-0034-1384578

27 Holm C, Mayr M, Hfter E et al. Perfusion Zones of the DIEP Flap Revisited: A Clinical Study. Plast Reconstr Surg 2006; 117: 37-43 Available from: http://content.wkhealth.com/linkback/openurl?sid=WKPTLP: landingpage \&an $=00006534-200601000-00006$

28 Wong C, Saint-Cyr M, Mojallal A et al. Perforasomes of the DIEP flap: vascular anatomy of the lateral versus medial row perforators and clinical implications. Plast Reconstr Surg 2010; 125: 772-782 Available from: http://www.ncbi.nlm.nih.gov/pubmed/20195105
29 Garvey PB, Clemens MW, Hoy AE et al. Muscle-sparing TRAM flap does not protect breast reconstruction from postmastectomy radiation damage compared with the DIEP flap. Plast Reconstr Surg 2014; 133: 223-233 Available from: http://www.ncbi.nlm.nih.gov/pubmed/24469158

30 Blondeel PN. Discussion: perfusion-related complications are similar for DIEP and muscle-sparing free TRAM flaps harvested on medial or lateral deep inferior epigastric artery branch perforators for breast reconstruction. Plast Reconstr Surg 2011; 128: 590e-592eAvailable from: http://www.ncbi.nlm.nih.gov/pubmed/22094756 